\title{
3. DATA REPORT: GEOCHEMICAL ANALYSES OF MASSIVE SULFIDE AND SEDIMENT SAMPLES FROM THE TAG HYDROTHERMAL MOUND ${ }^{1}$
}

\author{
D. Jay Miller ${ }^{2}$
}

\begin{abstract}
Bulk-rock geochemical analyses were performed on 29 samples of massive sulfide and sulfide-bearing sediment from the TAG hydrothermal mound. This pilot study was intended to determine whether or not enrichments of platinum group elements could be detected within the mound deposits. Although significant enrichments of Au were detected from near the surface of the mound and in at least one sample from within the mound, Pt concentrations were below the detection limit of the analytical method used. Pd concentrations in all samples were near or below detection limits as well. These data are included in this volume to complement other bulk-rock analyses and petrographic descriptions.
\end{abstract}

\section{INTRODUCTION}

Five different parts of the Trans-Atlantic Geotraverse (TAG) active hydrothermal mound were sampled during Leg 158 of the Ocean Drilling Program (ODP). The shorthand nomenclature for these locations adopted in Humphris, Herzig, Miller, et al. (1996) will be used in this report (see Fig. 1). This study was undertaken in order to assess the occurrence and distribution of platinum-group elements (PGE) within the TAG hydrothermal mound. Analysis of hydrothermal precipitates from the TAG mound has demonstrated a two to three orders of magnitude enrichment of Au relative to mid-ocean ridge basalt (Crocket, 1989, 1990; Hannington et al., 1991). Ravizza et al. (1996) report variable Os isotopic signatures for samples from the TAG mound, indicating that the Os in TAG sulfides could have been derived from either seawater or oceanic crust, or some combination of these two sources. Brügmann et al. (Chap. 7, this volume) also recognize a range in Os isotope compositions, but with the additional sample depth control afforded by drilling they interpret a stratigraphic correlation. Samples with the highest Os isotope ratios (indicating Os derived from seawater) are those recovered from near the upper part of the mound; samples with lower Os isotope ratios were recovered from deeper in the mound (indicating a contribution of Os from the underlying basalt). These data suggest that the hydrothermal fluids present at TAG are capable of leaching chalcophile elements from basalt and transporting these elements to sulfide deposits at the seafloor. Concentrations of PGE have not, however, been reported for samples dredged or collected by submersible from the TAG mound. The sampling strategy for this pilot study was to collect samples of $\mathrm{Cu}$-rich sulfides from various horizons within the mound to determine if there were subsurface concentrations of PGE.

\section{SAMPLE DESCRIPTIONS}

Because Pt and Pd are chalcophile elements commonly associated with sulfide minerals, samples were selected for analysis that were representative of the main sulfide-bearing mineralogies sampled. The terminology used to describe the major lithologic units is the same as used during Leg 158 (Humphris, Herzig, Miller, et al., 1996). Particular care was taken to sample where concentrations of chalcopyrite

${ }^{1}$ Herzig, P.M., Humphris, S.E., Miller, D.J., and Zierenberg, R.A. (Eds.), 1998. Proc. ODP, Sci. Results, 158: College Station, TX (Ocean Drilling Program).

${ }^{2}$ Ocean Drilling Program, Texas A\&M University Research Park, 1000 Discovery Drive, College Station, TX 77845, U.S.A. Jay_Miller@odp.tamu.edu

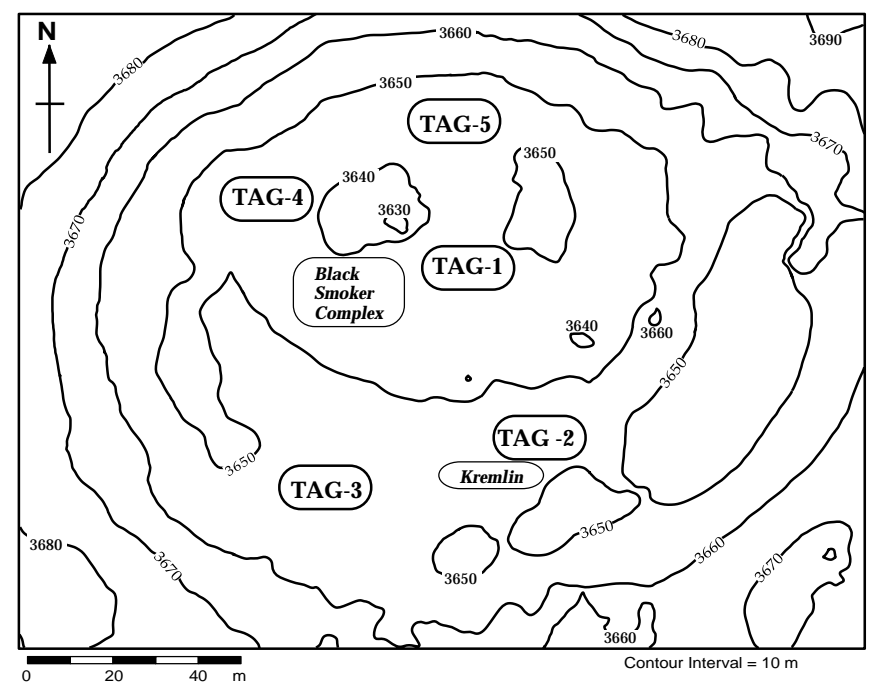

Figure 1. Sketch bathymetry map of the active TAG hydrothermal mound derived from $120 \mathrm{kHz}$, side-scan sonar data (Kleinrock et al., 1996). This map shows the general position of the five locations sampled during Leg 158.

were high (relative to the ubiquitous pyrite) and gangue minerals (anhydrite and silica) were low. Therefore, even samples named pyritesilica-anhydrite breccia were taken from the most sulfide-rich, but silica- and anhydrite-poor, parts of the core. In the pilot study, 20 samples of porous to massive sulfide and sulfide-bearing breccias and sands were analyzed. Table 1 summarizes petrographic observations from these samples. After determining that these samples were barren (or nearly so) of platinum-group elements at the analytical threshold, but recognizing that samples from the shallowest cores had elevated Au contents, another nine samples taken from within $\sim 30$ $\mathrm{cm}$ of the tops of cores were analyzed for $\mathrm{Au}, \mathrm{Pt}$, and $\mathrm{Pd}$. Two of these samples were of sufficient volume to analyze for major element oxides and trace elements as well.

\section{ANALYTICAL METHODS}

For bulk-rock analysis of major element oxides and some trace elements, an aliquot of the powdered sample was prepared using $\mathrm{LiBO}_{2}$ fusion (at $1025^{\circ} \mathrm{C}$ for $25 \mathrm{~min}$ ), followed by acid digestion $\left(\mathrm{HNO}_{3}\right)$ and analysis by an inductively coupled plasma emission spectrograph (ICP-ES). Two duplicate analyses were run to monitor analytical pre- 
Table 1. Petrographic summaries of massive sulfide and sulfide breccias.

\begin{tabular}{|c|c|}
\hline $\begin{array}{l}\text { Core, section, } \\
\text { interval top }(\mathrm{cm})\end{array}$ & Petrographic summary \\
\hline 957B-1R-2, 16 & Massive granular pyrite. $97 \%$ pyrite, $3 \%$ silica. Aggregates of medium to coarse euhedral grains. \\
\hline $957 \mathrm{C}-7 \mathrm{~N}-2,68$ & $\begin{array}{l}\text { Pyrite-silica-anhydrite breccia. } 55 \% \text { pyrite, } 35 \% \text { anhydrite, } 15 \% \text { silica, } 5 \% \text { chalcopyrite. Fine-grained, clast-supported breccia with nodular pyrite embedded in } \\
\text { an anhydrite matrix with subordinate gray chert. }\end{array}$ \\
\hline $957 \mathrm{C}-11 \mathrm{~N}-1,32$ & $\begin{array}{l}\text { Pyrite-anhydrite breccia. } 75 \% \text { pyrite, } 15 \% \text { anhydrite, } 10 \% \text { chalcopyrite. Chalcopyrite is concentrated near anhydrite veins. Pyrite is fine grained, with some } \\
\text { rounded grains and aggregates. }\end{array}$ \\
\hline $957 \mathrm{C}-11 \mathrm{~N}-3,116$ & $\begin{array}{l}\text { Pyrite-silica-anhydrite breccia. } 40 \% \text { pyrite, } 25 \% \text { silica, } 25 \% \text { anhydrite, } 10 \% \text { chalcopyrite. Pyrite has seriate grain-size distribution, fine to medium coarse grained. } \\
\text { Chalcopyrite is concentrated along vein selvage. Small altered basalt clasts are present. }\end{array}$ \\
\hline 957C-12N-2, 91 & Pyrite-anhydrite breccia. $50 \%$ pyrite, $30 \%$ anhydrite, $20 \%$ chalcopyrite. Nodular fine-grained clasts of pyrite. Chalcopyrite is concentrated along anhydrite veins. \\
\hline $957 \mathrm{C}-13 \mathrm{~N}-1,134$ & $\begin{array}{l}\text { Pyrite-silica-anhydrite breccia. } 70 \% \text { pyrite, } 15 \% \text { anhydrite, } 5 \% \text { silica, } 10 \% \text { chalcopyrite. Fine-grained pyrite in matrix of anhydrite and silica. Chalcopyrite is } \\
\text { most abundant along anhydrite veins. }\end{array}$ \\
\hline $957 \mathrm{C}-14 \mathrm{~N}-1,43$ & Massive pyrite-chalcopyrite. $55 \%$ pyrite, $30 \%$ chalcopyrite, $15 \%$ anhydrite. Fine-grained pyrite and chalcopyrite with thin anhydrite veins. \\
\hline $957 \mathrm{C}-16 \mathrm{~N}-2,91$ & $\begin{array}{l}\text { Silicified wallrock breccia. } 55 \% \text { pyrite, } 35 \% \text { silica, } 5 \% \text { anhydrite, } 5 \text { chalcopyrite. Silicified basalt fragments with abundant fine-grained euhedral pyrite. Porous } \\
\text { clast-supported breccia. }\end{array}$ \\
\hline 957E-4R-1, 5 & $\begin{array}{l}\text { Silicified wallrock breccia. } 45 \% \text { pyrite, } 20 \% \text { silica, } 15 \% \text { anhydrite, } 20 \% \text { chalcopyrite. Anhydrite and chalcopyrite along vein selvage. Pyritized and silicified } \\
\text { basalt fragments are common. }\end{array}$ \\
\hline $957 \mathrm{~F}-1 \mathrm{~N}-1,59$ & $\begin{array}{l}\text { Pyrite-anhydrite breccia. } 70 \% \text { pyrite, } 15 \% \text { anhydrite, } 10 \% \text { chalcopyrite, } 5 \% \text { silica. Angular, medium-sized grains of chalcopyrite with variably sized pyrite (fine } \\
\text { to coarse) grains in a clast-supported breccia with anhydrite matrix. }\end{array}$ \\
\hline 957G-1N-1, 23 & $\begin{array}{l}\text { Massive pyrite. } 85 \% \text { pyrite, } 5 \% \text { chalcopyrite, } 5 \% \text { anhydrite, } 5 \% \text { silica. Medium-grained, euhedral to colloform habit, a few percent of pore space is present. Silica } \\
\text { and anhydrite appear to be filling pore spaces, may be very late filling. }\end{array}$ \\
\hline $957 \mathrm{G}-3 \mathrm{~N}-1,25$ & $\begin{array}{l}\text { Pyrite breccia. } 85 \% \text { pyrite, } 5 \% \text { chalcopyrite, } 5 \% \text { anhydrite, } 5 \% \text { silica. Medium-grained, granular pyrite with relatively abundant chalcopyrite grains. Clast- } \\
\text { supported breccia with silica matrix. Anhydrite is in thin veins. }\end{array}$ \\
\hline $957 \mathrm{H}-5 \mathrm{~N}-1,40$ & $\begin{array}{l}\text { Pyrite-silica breccia. } 75 \% \text { pyrite, } 15 \% \text { silica, } 5 \% \text { anhydrite, } 5 \% \text { chalcopyrite. Rounded nodules of altered basalt that are now a mixture of gray silica and pyrite. } \\
\text { Pyrite also occurs as rounded nodules. Chalcopyrite is embedded in pyrite nodules. Anhydrite is in veins and vugs. }\end{array}$ \\
\hline $957 \mathrm{H}-8 \mathrm{~N}-1,1$ & Pyrite-silica breccia. $80 \%$ pyrite, $20 \%$ silica. Rounded to angular pyrite and pyritized basalt fragments in a silica matrix. Clast-supported breccia. \\
\hline $957 \mathrm{~K}-2 \mathrm{~N}-1,28$ & $\begin{array}{l}\text { Pyrite-silica-anhydrite breccia. } 70 \% \text { pyrite, } 20 \% \text { anhydrite, } 5 \% \text { chalcopyrite, } 5 \% \text { silica. Rounded to subangular pyrite-chalcopyrite clasts in clast-supported } \\
\text { breccia. Voids filled with anhydrite and amorphous silica. }\end{array}$ \\
\hline 957M-1R-2, 30 & $\begin{array}{l}\text { Pyrite-silica breccia. } 60 \% \text { pyrite, } 35 \% \text { silica, } 5 \% \text { chalcopyrite. Medium- to coarse-grained aggregates of pyrite occurring in altered basalt clasts and in matrix. } \\
\text { Chalcopyrite is fine grained and disseminated with some accumulation along margins of altered basalt clasts. }\end{array}$ \\
\hline $957 \mathrm{M}-5 \mathrm{R}-1,81$ & $\begin{array}{l}\text { Silicified wallrock breccia. } 73 \% \text { pyrite, } 25 \% \text { silica, } 2 \% \text { chalcopyrite. Most pyrite-chalcopyrite-rich fragment of this piece. Fine-grained, disseminated pyrite in } \\
\text { altered basalt clasts. }\end{array}$ \\
\hline 957P-1R-1, 1 & $\begin{array}{l}\text { Pyrite-anhydrite breccia. } 50 \% \text { pyrite, } 40 \% \text { anhydrite, } 10 \% \text { chalcopyrite. Fine, euhedral pyrite grains and rounded aggregates. Anhydrite in veins and in matrix of } \\
\text { clast-supported breccia. Chalcopyrite abundant on vein selvages. }\end{array}$ \\
\hline 957P-4R-1, 5 & $\begin{array}{l}\text { Massive pyrite. } 85 \% \text { pyrite, } 5 \% \text { anhydrite, } 5 \% \text { silica, } 5 \% \text { chalcopyrite. Anhydrite and silica in vugs and fractures. Pyrite is euhedral and fine grained, with } \\
\text { disseminated chalcopyrite. }\end{array}$ \\
\hline 957P-11R-1, 42 & $\begin{array}{l}\text { Pyrite-silica breccia. } 80 \% \text { pyrite, } 10 \% \text { silica, } 5 \% \text { anhydrite, } 5 \% \text { chalcopyrite. Pyrite is fine grained, disseminated in altered basalt clasts and in aggregates in silica } \\
\text { matrix of clast-supported breccia. Anhydrite is in voids and vugs. Chalcopyrite is disseminated. }\end{array}$ \\
\hline
\end{tabular}

cision, one selected by the laboratory, and a second included as an additional unknown (see Tables 2, 3, and 4). A well-characterized laboratory standard material was also analyzed to evaluate analytical accuracy. These data are in Table 2 . For trace-element analysis, a fouracid $\left(\mathrm{HNO}_{3}, \mathrm{HClO}_{4}, \mathrm{HF}, \mathrm{HCl}\right)$ digestion was used, followed by ICPES analysis (Table 3). Ba and $\mathrm{Sr}$ were analyzed after both digestion methods, and values are included in Tables 2 and 3 for comparison. Loss on ignition (LOI) was determined by igniting a 1-g sample split at $950^{\circ} \mathrm{C}$ for 90 minutes and measuring weight loss. PGE analyses generally require processing large quantities of sample $( \pm 1 \mathrm{~kg})$ in order to avoid unrepresentative analyses wherein the inclusion of a single PGE-bearing mineral grain in a small sample can bias the result. ODP sampling limitations precluded processing samples of this size, therefore the quantities homogenized for this study were on the order of 30 to $50 \mathrm{~g}$. Au, Pt, and Pd concentrations were determined by fusion digestion of $30 \mathrm{~g}$ of sample, lead-collection fire assay, and analysis by ICP-ES (Table 4). This data set is provided to complement other bulk rock analytical data elsewhere in this volume. Analyses were performed by Acme Analytical Laboratories, Vancouver, B.C.

\section{RESULTS}

Samples analyzed in this study had Pt and Pd contents at or below detection limits. Significant Au concentrations were analyzed in several samples. Most of the Au-bearing samples were from the uppermost cores taken from the mound, the significant exception was $\sim 700$ ppb taken from a depth of 21 meters below seafloor (mbsf; Sample $158-957 \mathrm{G}-3 \mathrm{~N}-1,25 \mathrm{~cm}$ ). A second series of analyses was performed on sulfide-bearing sands from uppermost parts of the core from the TAG-2, TAG-3, and TAG-4 locations. TAG-2 and TAG-3 samples all show elevated $\mathrm{Au}$ contents, but are still at or below detection limits for Pt and Pd. TAG-4 samples have only background levels of Au.

\section{ACKNOWLEDGMENTS}

The author thanks Editorial Review Board member Rob Zierenberg and an anonymous reviewer for their helpful comments. This work was supported by USSSP grant \#158-20911B.

\section{REFERENCES}

Crocket, J.H., 1989. PGE/Au fractionation in mid-ocean ridge volcanism. Progr. with Abstr., Geol. Assoc. Can., 14:70.

1990. Noble metals in seafloor hydrothermal mineralization from the Juan de Fuca and Mid-Atlantic ridges: a fractionation of gold from platinum metals in hydrothermal fluids. Can. Mineral., 28:639-648.

Hannington, M.D., Herzig, P.M., Scott, S.D., Thompson, G., and Rona, P.A., 1991. Comparative mineralogy and geochemistry of gold-bearing sulfide deposits on the mid-ocean ridges. Mar. Geol., 101:217-248.

Humphris, S.E., Herzig, P.M., Miller, D.J., et al., 1996. Proc. ODP, Init. Repts., 158: College Station, TX (Ocean Drilling Program).

Kleinrock, M.C., Humphris, S.E., and the Deep-TAG Team, 1996. Detailed structure and morphology of the TAG active hydrothermal mound and its geotectonic environment. In Humphris, S.E., Herzig, P.M., Miller, D.J., et al., Proc. ODP, Init. Repts., 158: College Station, TX (Ocean Drilling Program), 15-21.

Ravizza, G., Martin, C.E., German, C.R., and Thompson, G., 1996. Os isotopes as tracers in seafloor hydrothermal systems: metalliferous deposits from the TAG-hydrothermal area, $26^{\circ} \mathrm{N}$ Mid-Atlantic Ridge. Earth. Planet. Sci. Lett., 138:105-119.

Date of initial receipt: 3 June 1996

Date of acceptance: 21 February 1997

Ms 158SR-204 
Table 2. Geochemical analyses of samples from the TAG hydrothermal mound.

\begin{tabular}{|c|c|c|c|c|c|c|c|c|c|c|c|c|c|c|c|c|c|}
\hline \multirow{2}{*}{$\begin{array}{l}\text { Core, section, } \\
\text { interval }(\mathrm{cm})\end{array}$} & \multicolumn{2}{|c|}{$\begin{array}{r}\text { Component: } \\
\text { Detection limit*: }\end{array}$} & \multirow[t]{2}{*}{$\begin{array}{c}\mathrm{SiO}_{2} \\
0.01 \% \\
\end{array}$} & \multirow[t]{2}{*}{$\begin{array}{c}\mathrm{TiO}_{2} \\
0.01 \% \\
\end{array}$} & \multirow[t]{2}{*}{$\begin{array}{l}\mathrm{Al}_{2} \mathrm{O}_{3} \\
0.01 \% \\
\end{array}$} & \multirow[t]{2}{*}{$\begin{array}{l}\mathrm{Fe}_{2} \mathrm{O}_{3}{ }^{\mathrm{T}} \\
0.01 \% \\
\end{array}$} & $\begin{array}{l}\mathrm{MnO} \\
0.01 \% \\
\end{array}$ & $\begin{array}{l}\mathrm{MgO} \\
0.01 \% \\
\end{array}$ & $\begin{array}{c}\mathrm{CaO} \\
0.01 \% \\
\end{array}$ & $\begin{array}{l}\mathrm{Na}_{2} \mathrm{O} \\
0.01 \%\end{array}$ & $\begin{array}{c}\mathrm{K}_{2} \mathrm{O} \\
0.04 \% \\
\end{array}$ & $\begin{array}{c}\mathrm{P}_{2} \mathrm{O}_{5} \\
0.01 \% \\
\end{array}$ & $\begin{array}{c}\mathrm{S}^{\mathrm{T}} \\
0.1 \%\end{array}$ & $\begin{array}{l}\text { LOI } \\
0.1 \% \\
\end{array}$ & \multirow[t]{2}{*}{$\begin{array}{c}\mathrm{Ba} \\
5 \mathrm{ppm} \\
\end{array}$} & \multirow[t]{2}{*}{$\begin{array}{c}\mathrm{Sr} \\
10 \mathrm{ppm}\end{array}$} & $\begin{array}{c}\mathrm{Zr} \\
10 \mathrm{ppm} \\
\end{array}$ \\
\hline & Rock type & Location & & & & & & & & & & & & & & & \\
\hline 957B-1R-2, 16 & Massive pyrite & TAG-2 & 2.84 & 0.03 & 0.85 & 63.27 & bdl & 0.01 & 0.13 & 0.16 & 0.04 & 0.14 & 53.4 & 33.1 & 9 & bdl & 60 \\
\hline $957 \mathrm{C}-7 \mathrm{~N}-2,68$ & Pyrite-silica-anhydrite breccia & TAG-1 & 7.36 & 0.04 & 0.28 & 43.23 & bdl & 0.01 & 14.27 & 0.20 & bdl & 0.22 & 44.0 & 31.0 & 17 & 542 & 47 \\
\hline \multirow{3}{*}{$957 \mathrm{C}-11 \mathrm{~N}-3,116$} & Pyrite-anhydrite breccia & TAG-1 & 1.08 & bdl & 0.29 & 55.19 & bdl & bdl & 6.46 & 0.04 & 0.05 & 0.12 & 45.3 & 28.5 & 6 & 258 & bdl \\
\hline & Pyrite-silica-anhydrite breccia & TAG-1 & 24.64 & 0.15 & 0.88 & 36.64 & bdl & 0.18 & 9.09 & 0.09 & 0.04 & 0.20 & 33.2 & 24.1 & 16 & 397 & bdl \\
\hline & Split included as additional unknown & & 24.35 & 0.13 & 0.80 & 36.75 & bdl & 0.16 & 8.85 & 0.09 & 0.04 & 0.19 & 33.1 & 24.2 & 18 & 405 & bdl \\
\hline $957 \mathrm{C}-12 \mathrm{~N}-2,91$ & $\begin{array}{l}\text { Pyrite-anhydrite breccia } \\
\text { Pa }\end{array}$ & TAG-1 & 1.06 & bdl & 0.41 & 40.37 & bdl & 0.23 & 11.39 & bdl & bdl & 0.21 & 44.5 & 29.4 & bdl & 443 & bdl \\
\hline $957 \mathrm{C}-13 \mathrm{~N}-1,134$ & Pyrite-silica-anhydrite breccia & TAG-1 & 3.11 & 0.01 & 0.24 & 50.10 & bdl & 0.08 & 6.46 & bdl & 0.04 & 0.14 & 51.6 & 29.2 & 25 & 275 & 17 \\
\hline $957 \mathrm{C}-14 \mathrm{~N}-1,43$ & Massive pyrite-chalcopyrite & TAG-1 & 1.30 & 0.02 & 0.36 & 41.41 & bdl & 0.20 & 5.87 & bdl & bdl & 0.37 & 41.1 & 23.5 & 19 & 214 & bdl \\
\hline $957 \mathrm{C}-16 \mathrm{~N}-2,91$ & Silicified wallrock breccia & TAG-1 & 32.90 & 0.17 & 1.66 & 39.84 & bdl & 0.25 & 2.15 & 0.09 & 0.05 & 0.19 & 35.8 & 21.8 & 18 & 99 & 32 \\
\hline $957 \mathrm{E}-4 \mathrm{R}-1,5$ & Silicified wallrock breccia & TAG-1 & 19.31 & 0.04 & 0.31 & 41.92 & bdl & 0.09 & 5.93 & bdl & 0.04 & 0.32 & 37.0 & 22.7 & 17 & 252 & 34 \\
\hline 9 & Pyrite-anhydrite breccia & TAG-1 & $\begin{array}{r}19.31 \\
6.05\end{array}$ & 0.04 & 0.48 & 50.07 & $\begin{array}{l}\text { bdl } \\
\text { bal }\end{array}$ & 0.15 & 5.73 & $\begin{array}{l}\mathrm{Dal} \\
0.01\end{array}$ & $\begin{array}{l}0.04 \\
0.04\end{array}$ & 0.24 & 48.3 & 31.2 & 8 & 282 & $\begin{array}{l}34 \\
29\end{array}$ \\
\hline $957 \mathrm{G}-1 \mathrm{~N}-1,23$ & Massive pyrite & TAG-1 & 6.01 & bdl & 0.25 & 57.92 & bdl & 0.02 & 1.36 & bdl & bdl & 0.11 & 52.6 & 31.1 & 12 & 61 & 41 \\
\hline $957 \mathrm{G}-3 \mathrm{~N}-1,25$ & Pyrite breccia & TAG-1 & 1.61 & 0.04 & 0.20 & 61.23 & bdl & 0.03 & 1.08 & bdl & bdl & 0.08 & 55.9 & 32.2 & bdl & 58 & 13 \\
\hline $957 \mathrm{H}-5 \mathrm{~N}-1,40$ & Pyrite-silica breccia & TAG-2 & 16.68 & 0.12 & 1.01 & 48.09 & bdl & 0.36 & 3.41 & 0.19 & 0.16 & 0.49 & 46 & 27 & 57 & 69 & 42 \\
\hline $957 \mathrm{H}-8 \mathrm{~N}-1,1$ & Pyrite-silica breccia & TAG-2 & 18.03 & 0.07 & 0.55 & 52.21 & bdl & 0.05 & 0.43 & bdl & 0.04 & 0.13 & 45.7 & 28.2 & 6 & 25 & 215 \\
\hline $957 \mathrm{~K}-2 \mathrm{~N}-1,28$ & Pyrite-silica-anhydrite breccia & TAG-4 & 6.79 & 0.04 & 0.32 & 48.81 & 0.01 & 0.03 & 8.90 & bdl & 0.04 & 0.28 & 49.7 & 30.9 & bdl & 496 & 76 \\
\hline \multirow{3}{*}{$957 \mathrm{M}-5 \mathrm{R}-1,81$} & Pyrite-silica breccia & TAG-4 & 30.72 & 0.18 & 0.82 & 42.74 & bdl & 0.06 & 2.60 & 0.05 & 0.04 & 0.09 & 39.2 & 21.0 & 44 & 149 & 68 \\
\hline & Silicified wallrock breccia & TAG-4 & 25.17 & 0.19 & 1.68 & 46.55 & bdl & 0.07 & 0.31 & 0.17 & 0.06 & 0.08 & 41.7 & 24.6 & 47 & 27 & 61 \\
\hline & Laboratory-selected repeat analysis & & 24.81 & 0.16 & 1.64 & 46.47 & bdl & 0.05 & 0.29 & 0.18 & 0.06 & 0.05 & 40.8 & 24.5 & 32 & 26 & 59 \\
\hline 957P-1R-1, 1 & Pyrite-anhydrite breccia & TAG-5 & 2.47 & 0.04 & 0.42 & 35.53 & bdl & 0.04 & 15.30 & 0.04 & 0.04 & 0.25 & 39.4 & 20.5 & 25 & 836 & 163 \\
\hline 957P-4R-1, 5 & Massive pyrite & TAG-5 & 7.50 & bdl & 0.44 & 58.04 & bdl & 0.02 & 1.80 & bdl & 0.04 & 0.20 & 53.2 & 29.4 & 20 & 93 & 30 \\
\hline 957P-11R-1, 42 & Pyrite-silica breccia & TAG-5 & 11.59 & 0.01 & 0.25 & 55.65 & bdl & 0.03 & 1.33 & bdl & bdl & 0.05 & 51.2 & 28.0 & bdl & 80 & 29 \\
\hline $957 \mathrm{Q}-1 \mathrm{R}-1,20$ & Sulfide-bearing sand & TAG-3 & 47.89 & 0.01 & 0.06 & 26.51 & 0.01 & 0.10 & 0.12 & 0.35 & 0.04 & bdl & 22.9 & 13.8 & 5 & 12 & bdl \\
\hline 957Q-1R-1, 30 & Sulfide-bearing sand & $\begin{array}{l}\text { 1AG-3 } \\
\text { TAG-3 }\end{array}$ & $\begin{array}{l}4.89 \\
23.99\end{array}$ & $\begin{array}{l}\text { bdl } \\
\text { bdl }-19\end{array}$ & 0.04 & 36.93 & 0.01 & 0.07 & 0.07 & 0.29 & $\begin{array}{l}.04 \\
\text { bdl }\end{array}$ & $\begin{array}{l}\text { Dal } \\
\text { bdl }\end{array}$ & 36.0 & 20.9 & 3 & 7 & bdl \\
\hline $957 \mathrm{Q}-1 \mathrm{R}-3,48$ & Sulfide-bearing sand & TAG-3 & 17.37 & 0.04 & 0.25 & 49.35 & bdl & 0.06 & 0.55 & 0.16 & 0.04 & 0.27 & 42.2 & 24.3 & 17 & 33 & 10 \\
\hline \multirow[t]{2}{*}{ Standard SO-15 } & As analyzed with this sample set & & 50.92 & 2.81 & 12.69 & 7.47 & 1.38 & 7.14 & 5.84 & 2.31 & 1.78 & 2.81 & & & 2176 & 380 & 760 \\
\hline & Average composition of this standard & & 49.24 & 1.64 & 12.73 & 7.23 & 1.36 & 7.19 & 5.83 & 2.41 & 1.97 & 2.79 & & & 2189 & 385 & 733 \\
\hline
\end{tabular}

Notes: * = provided by Acme Analytical Laboratories, using 95\% confidence limits; bdl = below detection limit. Single acid digestion used in sample preparation 
Table 3. Trace element analyses of samples from the TAG hydrothermal mound.

\begin{tabular}{|c|c|c|c|c|c|c|c|c|c|c|c|c|c|c|c|}
\hline \multirow{2}{*}{$\begin{array}{l}\text { Core, section, } \\
\text { interval }(\mathrm{cm})\end{array}$} & \multicolumn{2}{|c|}{$\begin{array}{l}\text { Component: } \\
\text { Detection limit*: }\end{array}$} & \multirow[t]{2}{*}{$\begin{array}{c}\mathrm{Cu} \\
2 \mathrm{ppm} \\
\end{array}$} & \multirow[t]{2}{*}{$\begin{array}{c}\mathrm{Zn} \\
2 \mathrm{ppm} \\
\end{array}$} & \multirow[t]{2}{*}{$\begin{array}{c}\mathrm{Pb} \\
5 \mathrm{ppm} \\
\end{array}$} & \multirow[t]{2}{*}{$\begin{array}{c}\mathrm{Cd} \\
0.4 \mathrm{ppm}\end{array}$} & $\begin{array}{c}\mathrm{Ag} \\
0.5 \mathrm{ppm}\end{array}$ & $\begin{array}{c}\mathrm{Ni} \\
2 \mathrm{ppm} \\
\end{array}$ & $\begin{array}{c}\text { Mo } \\
2 \mathrm{ppm}\end{array}$ & $\begin{array}{c}\text { Co } \\
2 \mathrm{ppm}\end{array}$ & $\begin{array}{c}\mathrm{Mn} \\
5 \mathrm{ppm} \\
\end{array}$ & $\begin{array}{c}\mathrm{Fe} \\
0.01 \%\end{array}$ & $\begin{array}{c}\text { As } \\
5 \mathrm{ppm}\end{array}$ & $\begin{array}{c}\mathrm{U} \\
10 \mathrm{ppm} \\
\end{array}$ & $\begin{array}{c}\text { Th } \\
2 \mathrm{ppm}\end{array}$ \\
\hline & Rock type & Location & & & & & & & & & & & & & \\
\hline 957B-1R-2, 16 & Massive pyrite & TAG-2 & 592 & 36 & 29 & bdl & 1.0 & 7 & 60 & 216 & 63 & 42.68 & bdl & 18 & 3 \\
\hline $957 \mathrm{C}-7 \mathrm{~N}-2,68$ & Pyrite-silica-anhydrite breccia & TAG-1 & 20,037 & 235 & 27 & 0.9 & 1.3 & 12 & 48 & 139 & bdl & 26.17 & bdl & bdl & 3 \\
\hline $957 \mathrm{C}-11 \mathrm{~N}-1,32$ & Pyrite-anhydrite breccia & TAG-1 & 34,395 & 905 & 26 & 2.5 & 1.4 & 3 & 88 & 109 & 26 & 34.60 & bdl & 11 & bdl \\
\hline $957 \mathrm{C}-11 \mathrm{~N}-3,116$ & Pyrite-silica-anhydrite breccia & TAG-1 & 26,876 & 162 & 13 & 1.7 & 0.8 & 10 & 35 & 206 & 5 & 22.33 & bdl & bdl & bdl \\
\hline & Split included as additional unknown & & 26,768 & 150 & 15 & 2.1 & 0.8 & 12 & 36 & 201 & 9 & 22.55 & bdl & bdl & bdl \\
\hline $957 \mathrm{C}-12 \mathrm{~N}-2,91$ & Pyrite-anhydrite breccia & TAG-1 & 73,683 & 312 & 8 & 5.0 & 1.7 & bdl & 58 & 155 & 47 & 27.68 & bdl & bdl & 2 \\
\hline $957 \mathrm{C}-13 \mathrm{~N}-1,134$ & Pyrite-silica-anhydrite breccia & TAG-1 & 38,223 & 289 & 24 & 1.8 & 1.2 & 4 & 76 & 167 & bdl & 33.64 & bdl & bdl & 2 \\
\hline $957 \mathrm{C}-14 \mathrm{~N}-1,43$ & Massive pyrite-chalcopyrite & TAG-1 & $>100,000$ & 254 & bdl & 9.3 & 1.6 & bdl & 66 & 114 & 10 & 30.38 & bdl & bdl & 3 \\
\hline $957 \mathrm{C}-16 \mathrm{~N}-2,91$ & Silicified wallrock breccia & TAG-1 & 10,873 & 67 & 8 & bdl & bdl & 16 & 30 & 459 & 19 & 26.31 & bdl & bdl & bdl \\
\hline $957 \mathrm{E}-4 \mathrm{R}-1,5$ & Silicified wallrock breccia & TAG-1 & 66,752 & 134 & bdl & 4.8 & 1.7 & bdl & 73 & 168 & 29 & 26.66 & bdl & 12 & 5 \\
\hline $957 \mathrm{~F}-1 \mathrm{~N}-1,59$ & Pyrite-anhydrite breccia & TAG-1 & 38,481 & 434 & 18 & 0.9 & 2.9 & 5 & 68 & 290 & 11 & 31.73 & bdl & 13 & 2 \\
\hline $957 \mathrm{G}-1 \mathrm{~N}-1,23$ & Massive pyrite & TAG-1 & 20,708 & 853 & 28 & 1.4 & 1.2 & 2 & 66 & 97 & 22 & 38.19 & bdl & 10 & bdl \\
\hline $957 \mathrm{G}-3 \mathrm{~N}-1,25$ & Pyrite breccia & TAG-1 & 20,037 & 3,309 & 73 & 6.9 & 11.4 & 10 & 66 & 169 & 16 & 40.47 & 13 & 25 & bdl \\
\hline $957 \mathrm{H}-5 \mathrm{~N}-1,40$ & Pyrite-silica breccia & TAG-2 & 11,968 & 254 & 17 & 1.1 & 0.7 & 12 & 43 & 364 & 28 & 33.79 & bdl & bdl & bdl \\
\hline $957 \mathrm{H}-8 \mathrm{~N}-1,1$ & Pyrite-silica breccia & TAG-2 & 5,462 & 1,025 & 55 & 1.2 & 2.9 & 8 & 67 & 315 & 16 & 35.63 & 5 & bdl & 3 \\
\hline $957 \mathrm{~K}-2 \mathrm{~N}-1,28$ & Pyrite-silica-anhydrite breccia & TAG-4 & 24,434 & 308 & 26 & 1.8 & 2.1 & 7 & 52 & 134 & 19 & 30.07 & bdl & 15 & bdl \\
\hline $957 \mathrm{M}-1 \mathrm{R}-2,30$ & $\begin{array}{l}\text { Pyrite-silica breccia } \\
\text { a }\end{array}$ & TAG-4 & 9,897 & 289 & 22 & bdl & 1.5 & 12 & 32 & 227 & 26 & 27.45 & bdl & bdl & 2 \\
\hline $957 \mathrm{M}-5 \mathrm{R}-1,81$ & Silicified wallrock breccia & TAG-4 & 7,646 & 4,262 & 42 & 8.0 & 3.1 & 12 & 60 & 253 & 9 & & bdl & 18 & 3 \\
\hline 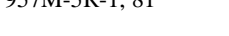 & Laboratory-selected repeat analysis & $1 \mathrm{AU}-4$ & 7,687 & $\begin{array}{r}4,242 \\
4,242\end{array}$ & 51 & $\begin{array}{l}0.0 \\
9.7\end{array}$ & 3.2 & 17 & 60 & 257 & 16 & 31.62 & bdl & $\begin{array}{l}10 \\
21\end{array}$ & bdl \\
\hline 957P-1R-1, 1 & Pyrite-anhydrite breccia & TAG-5 & 37,760 & 432 & bdl & 3.8 & 0.6 & 8 & 61 & 129 & bdl & 23.35 & bdl & bdl & 4 \\
\hline 957P-4R-1, 5 & Massive pyrite & TAG-5 & 15,296 & 610 & 50 & 3.6 & 2.2 & 7 & 70 & 207 & 34 & 36.91 & bdl & 40 & 4 \\
\hline 957P-11R-1, 42 & Pyrite-silica breccia & TAG-5 & 22,023 & 734 & 62 & 1.6 & 2.1 & 6 & 104 & 316 & 14 & 35.92 & bdl & 33 & bdl \\
\hline $957 \mathrm{Q}-1 \mathrm{R}-1,20$ & Sulfide-bearing sand & TAG-3 & 59,386 & 7,598 & 138 & 26.1 & 6.0 & 10 & 34 & 41 & 130 & 18.23 & 5 & bdl & bdl \\
\hline $957 \mathrm{Q}-1 \mathrm{R}-1,30$ & Sulfide-bearing sand & TAG-3 & 87,376 & 6,599 & 118 & 30.4 & 5.0 & 8 & 49 & 78 & 67 & 26.33 & bdl & bdl & bdl \\
\hline $957 \mathrm{Q}-1 \mathrm{R}-3,48$ & Sulfide-bearing sand & TAG-3 & 51,078 & 2,851 & 40 & 11.3 & 2.3 & 8 & 44 & 118 & 33 & 33.02 & 13 & 17 & bdl \\
\hline Standard CT/FA-100S & As analyzed with this sample set & & $\begin{array}{l}59 \\
59\end{array}$ & $\begin{array}{l}128 \\
148\end{array}$ & $\begin{array}{l}36 \\
40\end{array}$ & 16.2 & $\begin{array}{l}6.4 \\
60\end{array}$ & $\begin{array}{l}68 \\
76\end{array}$ & $\begin{array}{l}18 \\
20\end{array}$ & 29 & $\begin{array}{l}1170 \\
1176\end{array}$ & $\begin{array}{l}4.10 \\
4.37\end{array}$ & 28 & 22 & $\begin{array}{l}43 \\
41\end{array}$ \\
\hline
\end{tabular}

Notes: * = provided by Acme Analytical Laboratories, using 95\% confidence limits. bdl = below detection limit. Four-acid digestion used in sample preparation. 
Table 3 (continued).

\begin{tabular}{|c|c|c|c|c|c|c|c|c|c|c|c|c|c|c|c|c|}
\hline \multirow{2}{*}{$\begin{array}{l}\text { Core, section, } \\
\text { interval }(\mathrm{cm})\end{array}$} & \multicolumn{2}{|c|}{$\begin{array}{r}\text { Component: } \\
\text { Detection limit*: }\end{array}$} & \multirow[t]{2}{*}{$\begin{array}{c}\mathrm{Sr} \\
2 \mathrm{ppm}\end{array}$} & \multirow[t]{2}{*}{$\begin{array}{c}\mathrm{Sb} \\
5 \mathrm{ppm} \\
\end{array}$} & \multirow[t]{2}{*}{$\begin{array}{c}\mathrm{Bi} \\
5 \mathrm{ppm} \\
\end{array}$} & \multirow[t]{2}{*}{$\begin{array}{c}\mathrm{V} \\
2 \mathrm{ppm} \\
\end{array}$} & $\begin{array}{c}\mathrm{Ca} \\
0.01 \% \\
\end{array}$ & $\begin{array}{c}\mathrm{La} \\
2 \mathrm{ppm}\end{array}$ & $\begin{array}{c}\mathrm{Ba} \\
1 \mathrm{ppm} \\
\end{array}$ & $\begin{array}{c}\mathrm{Ti} \\
0.01 \% \\
\end{array}$ & $\begin{array}{c}\mathrm{Al} \\
0.01 \% \\
\end{array}$ & $\begin{array}{c}\mathrm{Na} \\
0.01 \% \\
\end{array}$ & $\begin{array}{c}\text { Sn } \\
2 \mathrm{ppm} \\
\end{array}$ & $\begin{array}{c}\mathrm{Nb} \\
2 \mathrm{ppm} \\
\end{array}$ & $\begin{array}{c}\mathrm{Be} \\
1 \mathrm{ppm} \\
\end{array}$ & $\begin{array}{c}\mathrm{Sc} \\
1 \mathrm{ppm}\end{array}$ \\
\hline & Rock type & Location & & & & & & & & & & & & & & \\
\hline 957B-1R-2, 16 & Massive pyrite & TAG-2 & 3 & 5 & 8 & 11 & 0.08 & bdl & 10 & bdl & 0.39 & 0.12 & 4 & 3 & 2 & bdl \\
\hline $957 \mathrm{C}-7 \mathrm{~N}-2,68$ & Pyrite-silica-anhydrite breccia & TAG-1 & 480 & bdl & 16 & 2 & 8.19 & bdl & 3 & bdl & 0.07 & 0.10 & 3 & bdl & bdl & bdl \\
\hline $957 \mathrm{C}-11 \mathrm{~N}-1,32$ & Pyrite-anhydrite breccia & TAG-1 & 237 & 9 & 27 & 3 & 3.88 & bdl & 4 & bdl & 0.11 & 0.04 & 3 & bdl & bdl & bdl \\
\hline \multirow{2}{*}{$957 \mathrm{C}-11 \mathrm{~N}-3,116$} & Pyrite-silica-anhydrite breccia & TAG-1 & 386 & 8 & 11 & 11 & 5.72 & bdl & 6 & 0.01 & 0.35 & 0.07 & 3 & bdl & 1 & 1 \\
\hline & Split included as additional unknown & & 393 & 7 & 14 & 15 & 5.65 & bdl & 3 & bdl & 0.35 & 0.06 & 3 & bdl & bdl & bdl \\
\hline $957 \mathrm{C}-12 \mathrm{~N}-2,91$ & Pyrite-anhydrite breccia & TAG-1 & 426 & 14 & 47 & 7 & 7.00 & bdl & 4 & bdl & 0.16 & 0.03 & 11 & bdl & bdl & bdl \\
\hline $957 \mathrm{C}-13 \mathrm{~N}-1,134$ & Pyrite-silica-anhydrite breccia & TAG-1 & 255 & bdl & 17 & 2 & 3.93 & bdl & 2 & bdl & 0.08 & 0.03 & 2 & bdl & bdl & bdl \\
\hline $957 \mathrm{C}-14 \mathrm{~N}-1,43$ & Massive pyrite-chalcopyrite & TAG-1 & 213 & 16 & 95 & 9 & 3.81 & bdl & 2 & bdl & 0.13 & 0.02 & 14 & bdl & bdl & bdl \\
\hline $957 \mathrm{C}-16 \mathrm{~N}-2,91$ & Silicified wallrock breccia & TAG-1 & 97 & bdl & 7 & 23 & 1.42 & bdl & 2 & 0.05 & 0.76 & 0.15 & bdl & 3 & 2 & 2 \\
\hline $957 \mathrm{E}-4 \mathrm{R}-1,5$ & Silicified wallrock breccia & TAG-1 & 243 & bdl & 34 & 2 & 3.73 & bdl & 2 & bdl & 0.09 & 0.02 & 6 & bdl & bdl & bdl \\
\hline $957 \mathrm{~F}-1 \mathrm{~N}-1,59$ & Pyrite-anhydrite breccia & TAG-1 & 248 & bdl & 17 & 10 & 3.26 & bdl & 2 & bdl & 0.19 & 0.03 & bdl & bdl & bdl & bdl \\
\hline $957 \mathrm{G}-1 \mathrm{~N}-1,23$ & Massive pyrite & TAG-1 & 56 & bdl & 10 & 16 & 0.85 & bdl & 1 & bdl & 0.09 & 0.02 & 2 & 2 & bdl & bdl \\
\hline $957 \mathrm{G}-3 \mathrm{~N}-1,25$ & Pyrite breccia & TAG-1 & 51 & 19 & 18 & 9 & 0.68 & bdl & 1 & bdl & 0.08 & 0.02 & 3 & bdl & bdl & bdl \\
\hline $957 \mathrm{H}-5 \mathrm{~N}-1,40$ & Pyrite-silica breccia & TAG-2 & 48 & bdl & 12 & 19 & 0.66 & bdl & 1 & 0.01 & 0.38 & 0.08 & 3 & 2 & 1 & 1 \\
\hline $957 \mathrm{H}-8 \mathrm{~N}-1,1$ & Pyrite-silica breccia & TAG-2 & 23 & bdl & 18 & 9 & 0.27 & bdl & bdl & bdl & 0.23 & 0.04 & 3 & bdl & bdl & bdl \\
\hline $957 \mathrm{~K}-2 \mathrm{~N}-1,28$ & Pyrite-silica-anhydrite breccia & TAG-4 & 439 & bdl & 11 & 8 & 5.09 & bdl & 5 & 0.01 & 0.11 & 0.04 & bdl & bdl & bdl & bdl \\
\hline 957M-1R-2, 30 & Pyrite-silica breccia & TAG-4 & 142 & 5 & bdl & 19 & 1.65 & bdl & 4 & 0.01 & 0.31 & 0.05 & bdl & 3 & bdl & bdl \\
\hline \multirow[t]{2}{*}{$957 \mathrm{M}-5 \mathrm{R}-1,81$} & Silicified wallrock breccia & TAG-4 & 19 & bdl & 5 & 28 & 0.14 & bdl & 36 & 0.06 & 0.74 & 0.14 & 3 & 4 & 3 & 3 \\
\hline & Laboratory-selected repeat analysis & & 18 & 14 & 9 & 29 & 0.13 & bdl & 32 & 0.07 & 0.76 & 0.15 & bdl & bdl & 8 & 3 \\
\hline 957P-1R-1, 1 & Pyrite-anhydrite breccia & TAG-5 & 763 & bdl & 20 & 8 & 9.13 & bdl & 6 & bdl & 0.14 & 0.06 & bdl & bdl & bdl & bdl \\
\hline 957P-4R-1, 5 & $\begin{array}{l}\text { Massive pyrite } \\
\text { litala }\end{array}$ & TAG-5 & 82 & bdl & 18 & 10 & 1.08 & bdl & bdl & bdl & 0.15 & 0.03 & bdl & bdl & bdl & bdl \\
\hline 957P-11R-1, 42 & Pyrite-silica breccia & TAG-5 & 73 & 5 & 17 & 2 & 0.80 & bdl & bdl & bdl & 0.06 & 0.02 & bdl & bdl & bdl & bdl \\
\hline 957Q-1R-1,20 & Sulfide-bearing sand & TAG-3 & 12 & 23 & 18 & 33 & 0.09 & bdl & 5 & bdl & 0.04 & 0.25 & 4 & bdl & 3 & bdl \\
\hline $957 \mathrm{Q}-1 \mathrm{R}-1,30$ & Sulfide-bearing sand & TAG-3 & 7 & 29 & 41 & 19 & 0.06 & bdl & 3 & bdl & 0.03 & 0.21 & 7 & bdl & bdl & bdl \\
\hline $957 \mathrm{Q}-1 \mathrm{R}-3,48$ & Sulfide-bearing sand & TAG-3 & 27 & 18 & 49 & 10 & 0.32 & bdl & bdl & bdl & 0.05 & 0.13 & 5 & bdl & bdl & bdl \\
\hline \multirow[t]{2}{*}{ Standard CT/FA-100S } & As analyzed with this sample set & & 235 & 27 & 18 & 100 & 1.08 & 43 & 917 & 0.34 & 6.23 & 1.44 & 17 & 7 & bdl & 13 \\
\hline & Average composition of this standard & & 239 & 22 & 17 & 135 & 1.16 & 45 & 832 & 0.33 & 7.27 & 1.66 & 19 & 8 & 2 & 15 \\
\hline
\end{tabular}


Table 4. Precious metal concentrations in samples from the TAG hydrothermal mound.

\begin{tabular}{|c|c|c|c|c|c|}
\hline \multirow{2}{*}{$\begin{array}{l}\text { Hole, core, section, } \\
\text { interval }(\mathrm{cm})\end{array}$} & \multicolumn{2}{|l|}{$\begin{array}{r}\text { Component: } \\
\text { Detection limit*: }\end{array}$} & \multirow[t]{2}{*}{$\begin{array}{c}\mathrm{Au} \\
2 \mathrm{ppb}\end{array}$} & \multirow[t]{2}{*}{$\begin{array}{c}\mathrm{Pt} \\
3 \mathrm{ppb}\end{array}$} & \multirow[t]{2}{*}{$\begin{array}{c}\mathrm{Pd} \\
3 \mathrm{ppb} \\
\end{array}$} \\
\hline & Rock type & Location & & & \\
\hline 957B-1R-1, 11 & Sulfide-bearing sand & TAG-2 & 2797 & bdl & bdl \\
\hline 957B-1R-1, 21 & Sulfide-bearing sand & TAG-2 & 1823 & bdl & bdl \\
\hline $957 \mathrm{~B}-1 \mathrm{R}-1,30$ & Sulfide-bearing sand & TAG-2 & 1909 & bdl & bdl \\
\hline $957 \mathrm{~B}-1 \mathrm{R}-2,16$ & Massive pyrite & TAG-2 & 71 & bdl & 5 \\
\hline 957C-7N-2, 68 & Pyrite-silica-anhydrite breccia & TAG-1 & 103 & bdl & 3 \\
\hline $957 \mathrm{C}-11 \mathrm{~N}-1,32$ & Pyrite-anhydrite breccia & TAG-1 & 72 & bdl & 4 \\
\hline \multirow[t]{2}{*}{$957 \mathrm{C}-11 \mathrm{~N}-3,116$} & Pyrite-silica-anhydrite breccia & TAG-1 & 48 & bdl & 3 \\
\hline & Split included as additional unknown & & 46 & bdl & 3 \\
\hline $957 \mathrm{C}-12 \mathrm{~N}-2,91$ & Pyrite-anhydrite breccia & TAG-1 & 83 & bdl & 4 \\
\hline $957 \mathrm{C}-13 \mathrm{~N}-1,134$ & Pyrite-silica-anhydrite breccia & TAG-1 & 46 & bdl & 3 \\
\hline $957 \mathrm{C}-14 \mathrm{~N}-1,43$ & Massive pyrite-chalcopyrite & TAG-1 & 43 & bdl & 5 \\
\hline $957 \mathrm{C}-16 \mathrm{~N}-2,91$ & Silicified wallrock breccia & TAG-1 & 14 & bdl & 4 \\
\hline $957 \mathrm{E}-4 \mathrm{R}-1,5$ & Silicified wallrock breccia & TAG-1 & 39 & bdl & 4 \\
\hline $957 \mathrm{~F}-1 \mathrm{~N}-1,59$ & Pyrite-anhydrite breccia & TAG-1 & 222 & bdl & 4 \\
\hline $957 \mathrm{G}-1 \mathrm{~N}-1,23$ & Massive pyrite & TAG-1 & 309 & bdl & 3 \\
\hline $957 \mathrm{G}-3 \mathrm{~N}-1,25$ & Pyrite breccia & TAG-1 & 699 & bdl & 4 \\
\hline $957 \mathrm{H}-5 \mathrm{~N}-1,40$ & Pyrite-silica breccia & TAG-2 & 128 & bdl & 5 \\
\hline $957 \mathrm{H}-8 \mathrm{~N}-1,1$ & Pyrite-silica breccia & TAG-2 & 193 & bdl & 3 \\
\hline $957 \mathrm{~K}-2 \mathrm{~N}-1,28$ & Pyrite-silica-anhydrite breccia & TAG-4 & 227 & bdl & 5 \\
\hline $957 \mathrm{M}-1 \mathrm{R}-1,9$ & Sulfide-bearing sand & TAG-4 & 93 & bdl & bdl \\
\hline 957M-1R-1, 21 & Sulfide-bearing sand & TAG-4 & 65 & bdl & bdl \\
\hline $957 \mathrm{M}-1 \mathrm{R}-1,33$ & Sulfide-bearing sand & TAG-4 & 24 & bdl & bdl \\
\hline $957 \mathrm{M}-1 \mathrm{R}-2,30$ & Pyrite-silica breccia & TAG-4 & 154 & bdl & 5 \\
\hline \multirow{2}{*}{$957 \mathrm{M}-5 \mathrm{R}-1,81$} & Silicified wallrock breccia & TAG-4 & 223 & bdl & 3 \\
\hline & Laboratory-selected repeat analysis & & 223 & bdl & 4 \\
\hline 957P-1R-1, 1 & Pyrite-anhydrite breccia & TAG-5 & 103 & bdl & bdl \\
\hline 957P-4R-1, 5 & Massive pyrite & TAG-5 & 189 & bdl & bdl \\
\hline 957P-11R-1, 42 & Pyrite-silica breccia & TAG-5 & 268 & bdl & 4 \\
\hline 957Q-1R-1, 20 & Sulfide-bearing sand & TAG-3 & 1171 & bdl & bdl \\
\hline 957Q-1R-1, 30 & Sulfide-bearing sand & TAG-3 & 1217 & bdl & bdl \\
\hline 957Q-1R-3, 48 & Sulfide-bearing sand & TAG-3 & 737 & bdl & 3 \\
\hline \multirow[t]{2}{*}{ Standard CT/FA-100S } & As analyzed with this sample set & & 44 & 46 & 45 \\
\hline & Average composition of this standard & & 49 & 46 & 46 \\
\hline
\end{tabular}

Note: $*$ = provided by Acme Analytical Laboratories, using 95\% confidence limits; bdl = below detection limit. 